\title{
Identification of anesthetic-induced expression changes using DNA microarray
}

\author{
ZAIQI YANG ${ }^{1,2}$, MENGYUAN ZHANG ${ }^{1}$, GONGMING WANG $^{1}$, PIHONG WEI $^{2}$ and SHENQIANG GAO ${ }^{2}$ \\ ${ }^{1}$ Department of Anesthesiology, Shandong Provincial Hospital, Shandong University, Jinan, Shandong 250012; \\ ${ }^{2}$ Department of Anesthesiology, Taian Central Hospital, Taian, Shandong 271000, P.R. China
}

Received February 9, 2014; Accepted August 29, 2014

DOI: $10.3892 / \mathrm{mmr} .2014 .2669$

\begin{abstract}
The present study aimed to identify changes in atrial gene expression induced by sevoflurane and propofol using DNA microarray. The expression profiles of GSE4386 in atrial samples, obtained from patients who had received either the anesthetic gas sevoflurane or the intravenous anesthetic propofol prior to and following off-pump coronary artery bypass graft $(\mathrm{CABG})$ surgery, were downloaded from the Gene Expression Omnibus database. The differentially expressed genes (DEGs) in the sevoflurane and the propofol groups were then identified and compared. Subsequently, a functional enrichment analysis was performed for the DEGs. The interactive functional modules for common, sevoflurane-specific and propofol-specific DEGs were then constructed for analysis of the biological processes. The percentages of common DEGs were 31.3 (275/879) and 94.8\% (275/290) in the sevoflurane group and propofol groups, respectively. The functional categories for the common, sevoflurane-specific and propofol-specific DEGs were similar. Overall, two, one, and one functional modules were identified for the common DEGs, propofol specific DEGs and sevoflurane specific DEGs, respectively. DEGs in the modules were involved in cellular processes, including the 'regulation of transcription' and 'regulation of cellular process', which were similar to the functional annotations for the DEGs. Therefore, sevoflurane and propofol may synergistically reduce myocardial reperfusion injury in patients undergoing off-pump CABG surgery.
\end{abstract}

\section{Introduction}

Coronary artery bypass grafting (CABG) surgery is a common cardiac surgical procedure. CABG surgery is performed either with or without cardiopulmonary bypass,

Correspondence to: Dr Mengyuan Zhang, Department of Anesthesiology, Shandong Provincial Hospital, Shandong University, 324 Jingwuweiqi Road, Jinan, Shandong 250012, P.R. China

E-mail: mengyuanzhang2013@hotmail.com

Key words: sevoflurane, propofol, coronary artery bypass grafting, differentially expressed genes referred to as the traditional method of on-pump CABG and the newer off-pump CABG, respectively (1). A number of studies have confirmed that, compared with on-pump CABG, off-pump CABG has a lower risk of renal damage, myocardial injury, brain injury, stroke, atrial fibrillation and neurocognitive and organ dysfunction (2-5). Clinical trials have revealed that off-pump CABG has no significant benefit regarding mortality, stroke or myocardial infarction compared with on-pump CABG, although off-pump CABG may be preferable when patients have contraindications for cardiopulmonary bypass $(6,7)$. CABG surgery can lead to ischemic injury due to a transient period of local ischemia with temporary occlusion of the target vessel, particularly in patients with poor cardiac contractile function (8-10). Certain cytokines, including interleukin (IL)- 6 , interferon- $\gamma$, high sensitivity $\mathrm{C}$-reactive protein and granulocyte colony-stimulating factor, are released in off-pump CABG surgery to a similar or larger extent than in on-pump CABG surgery $(11,12)$. Therefore, perioperative management for off-pump CABG surgery requires improvement to reduce myocardial ischemic-reperfusion damage.

Various interventions, including certain anesthetic agents, prior to and following myocardial ischemia have the potential, to a certain extent, to reduce myocardial ischemic damage and subsequent reperfusion injury $(13,14)$. Sevoflurane is a type of inhalational anaesthetic agent, which significantly reduces the size of infarcts and $\mathrm{Ca}^{2+}$ loading to protect the myocardium against reperfusion injury $(14,15)$ and has myocardial protective properties for low risk patients, who are undergoing CABG surgery (16). It has been demonstrated that preconditioning by sevoflurane downregulates platelet-endothelial cell adhesion molecule-1 and upregulates catalase in atrial biopsies from patients undergoing CABG surgery (17). The levels of IL-6 and IL-8 in the serum are suppressed by sevoflurane, while the IL-10 and IL-1 receptor antagonist, remain significantly increased in patients undergoing CABG (18). In addition to volatile anesthetics, intravenous anesthetics, including propofol, may also reduce myocardial reperfusion injury in patients undergoing CABG surgery (19). It has been observed that propofol reduces lipid peroxidation, which is mediated by free radicals, and systemic inflammation in patients with impaired left ventricular function undergoing CABG surgery, with no differences in urinary isoprostane concentrations 
or leucocyte function (20). The activities of nitric oxide synthase and phosphoinositide-3-kinase/Akt, which are maintained by propofol, may be partly responsible for reduced ischemic-reperfusion injury $(21,22)$. Kottenberg et al (23) revealed that propofol attenuates the effects of remote ischemic preconditioning in patients undergoing $\mathrm{CABG}$ surgery (23) and, with a chemical structure similar to that of free-radical scavengers, interferes with remote ischemic preconditioning, since the release of free radicals is necessary to evoke isoflurane-induced preconditioning (24-26). There is increasing evidence that sevoflurane and propofol have effective cardioprotective effects, however, the underlying mechanisms of these anaesthetic agents for cardioprotection remain to be elucidated (27). Lucchinetti et al (28) analyzed the differentially regulated pathways in sevoflurane- and propofol-treated patients by gene expression profiling using a gene set enrichment analysis method (28). In the present study, common differentially expressed genes (DEGs), propofol-specific and sevoflurane-specific DEGs, were identified. Subsequently, in addition to performing functional annotation of these DEGs, functional analysis of the same DEGs in the selected interactive functional modules was performed. The results of the present study aimed to reveal specific novel mechanisms underlying the cardioprotective effects of sevoflurane and propofol.

\section{Materials and methods}

Samples. The expression profiling of GSE4386 produced by Lucchinetti et al (28) and including 40 atrial samples, was downloaded from the National Center of Biotechnology Information Gene Expression Omnibus (http://www.ncbi. nlm.nih.gov/geo/), which was based on the Affymetrix Human Genome U133 Plus 2.0 Array platform (Affymetrix, Santa Clara, CA, USA).

The 40 atrial samples were collected at the beginning and at the end of the off-pump CABG surgery and included 20 atrial samples from 10 patients receiving the anesthetic gas sevoflurane (Sevorane; Abbott, Baar, Switzerland) and 20 atrial samples from 10 patients receiving the intravenous anesthetic propofol (Diprivan 2\%; AstraZeneca, Zug, Switzerland). The sevoflurane and propofol were adjusted to maintain the blood pressure and heart rate within $20 \%$ of the baseline values (28). The downloaded expression profiling for the 40 atrial samples were further analyzed to identify DEGs.

Data pre-treatment and DEG identification. The platform annotation file in txt format, provided by Affymetrix, was used to map the association between the probes and the gene symbols. Subsequently, quartile data normalization was performed using the Affy package in the R language (29) and the Multtest package (Bioconductor, Fred Hutchinson Cancer Research Center, Seattle, WA, USA; 30) was used to identify the genes differentially expressed between the samples at the beginning and at the end of surgery in the sevoflurane and propofol groups. The Benjamini-Hochberg procedure (31) was used to adjust the raw P-values into false discovery rate (FDR). FDR $<0.05$ and $\mid \log F C l>1$ were used as cut-off criteria in the DEG identification, where FC stands for fold change.
Intergroup comparison of DEGs. Subsequent to obtaining the DEGs in the sevoflurane and propofol groups, the sevoflurane specific, propofol-specific and common DEGs between the two groups were selected. The expression levels of the sevoflurane-specific, propofol-specific and common DEGs in the samples at the beginning of surgery were then compared with those in the samples at the end of surgery using a t-test. $\mathrm{P}<0.05$ was considered to indicate a statistically significant difference.

Functional enrichment analysis for DEGs. Gene ontology (GO) biological process enrichment analyses for the screened DEGs in the sevoflurane and propofol groups were performed using the Database for Annotation, Visualization and Integrated Discovery (DAVID, http://david.abcc.ncifcrf.gov/), containing analytical tools and bioinformatic resources for the systematic extraction of biological functions (32). FDR $<0.05$ was selected as a cut-off criterion. The annotated protein sequences of the DEGs were compared with the proteins in clusters of orthologous groups of proteins (COG; http://www. ncbi.nlm.nih.gov/COG) (33) database using the Basic Local Alignment Search tool (BLASTX) (34) with a cut-off threshold of $\mathrm{E}<1 \times 10^{-5}$. The common DEGs, propofol-specific DEGs and sevoflurane-specific DEGs were then classified into different functional annotations and COG categories, including cellular component (CC), molecular function (MF) and biological process (BP).

Analysis of interactive function modules for DEGs. WebGestalt software (Vanderbilt University, Nashville, TN, USA; http://bioinfo.vanderbilt.edu/webgestalt/; 35,36) was used to search for the interactive function modules of DEGs within the cut-off criterion of FDR $<0.05$. Analysis of the GO biological processes associated with the DEGs in the modules was then performed using Expression Analysis Systematic Explorer software (version 2.0, http://david.abcc.ncifcrf.gov/ ease/ease.jsp) using the threshold of $\mathrm{P}<0.05$.

\section{Results}

$D E G$ screening. The normalized expression profiles were analyzed to identify the DEGs with FDR $<0.05$ and $|\log F C|>1$. A total of 879 and 290 DEGs were selected in the sevoflurane and propofol groups, respectively.

DEG comparison. The DEGs in the sevoflurane group were compared with those in the propofol group. There were 275 common DEGs, 604 sevoflurane-specific DEGs and 15 propofol-specific DEGs (Fig. 1A). The percentages of the common DEGs were $31.3(275 / 879)$ and $94.8 \%(275 / 290)$ in the sevoflurane and propofol groups, respectively.

The differences in the expression levels were in accordance with the DEG identification (Fig. 1B). The sevoflurane-specific DEGs were differentially expressed in the sevoflurane group $(\mathrm{P}=0.01239)$ compared with the propofol group $(\mathrm{P}=0.214)$ and the propofol-specific DEGs were differentially expressed in the propofol group $(\mathrm{P}=0.02206)$ compared with the sevoflurane group $(\mathrm{P}=0.2243)$. Furthermore, the common DEGs were differentially expressed in the sevoflurane group $\left(\mathrm{P}=2.2 \times 10^{-16}\right)$ and propofol group $\left(\mathrm{P}=2.98 \times 10^{-14}\right)$. 
A

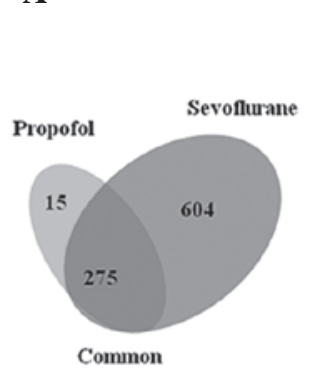

B

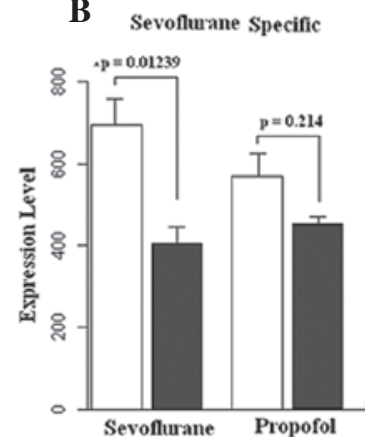

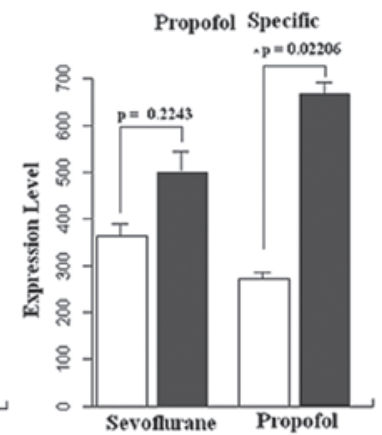

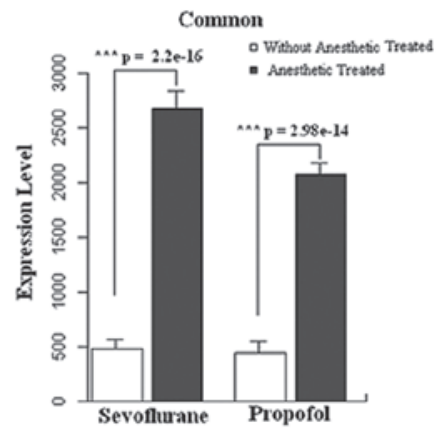

Figure 1. Comparision of DEGs between the sevoflurane group and the propofol group. (A) Differences in the expression levels of the common DEGs, sevoflurane-specific DEGs and propofol-specific DEGs. (B) White and black columns represent samples collected at the beginning and end of off-pump CABG surgery, respectively. DEG, differentially expressed genes.

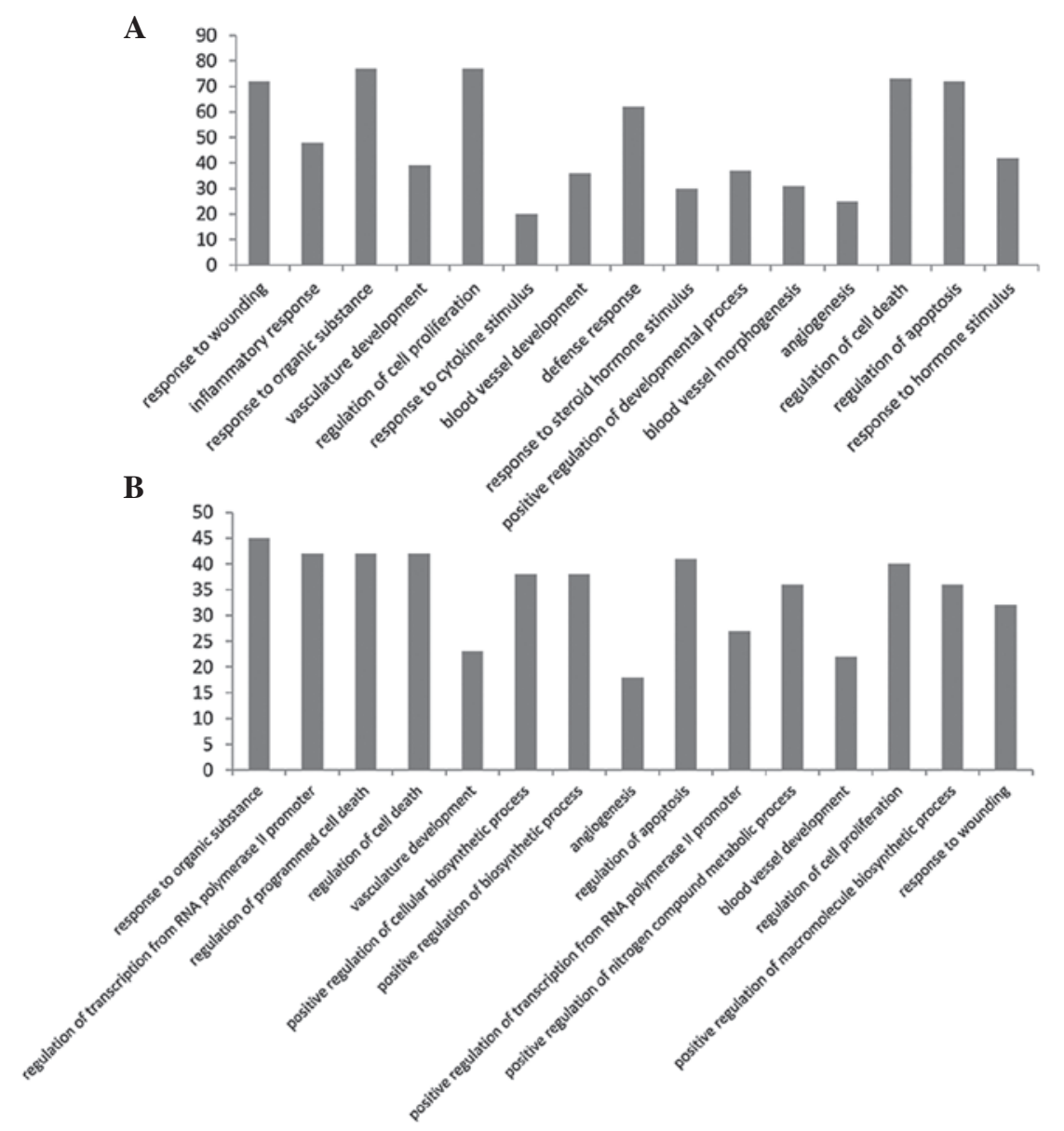

Figure 2. Gene ontology biological process enrichment analysis of the screened differentially expressed genes in the (A) sevoflurane and (B) propofol groups.

Functional categories for DEGs. The 'response to wounding' function, involving 72 DEGs, was identified as the most significant GO term of the 879 DEGs in the sevoflurane group and the 'response to organic substance' function, involving 45 DEGs, was the most significant GO term of the 290 DEGs in the propofol group (Fig. 2). The common DEGs, sevoflurane-specific DEGs and propofol-specific DEGs were analyzed using BLASTX by comparing with the COG database with the $\mathrm{E}<1 \mathrm{x} 10^{-5}$. The results demonstrated that the functional categories in $\mathrm{CC}, \mathrm{MF}$ and $\mathrm{BP}$ for the common, sevoflurane-specific and propofol-specific DEGs were similar (Fig.3).
Analysis of modules. Total functional modules of the common DEGs (two total), propofol-specific DEGs and sevoflurane-specific DEGs, with an FDR $<0.05$, were identified (Fig. 4). There were 10 and 18 DEGs in the two functional modules for the common DEGs, including activating transcription factor 3 (ATF3), jun D proto-oncogene (JUND), mitogen-activated protein kinase (MAPK)3, FBJ murine osteosarcoma viral oncogene homolog B (FOSB), jun B proto-oncogene (JUNB) and tumor necrosis factor $\alpha$-induced protein 3 (TNF $\alpha$ IP3). A total of two upregulated DEGs, including CD93 and leucine rich repeat containing 32 (LRRC32) were involved 
A

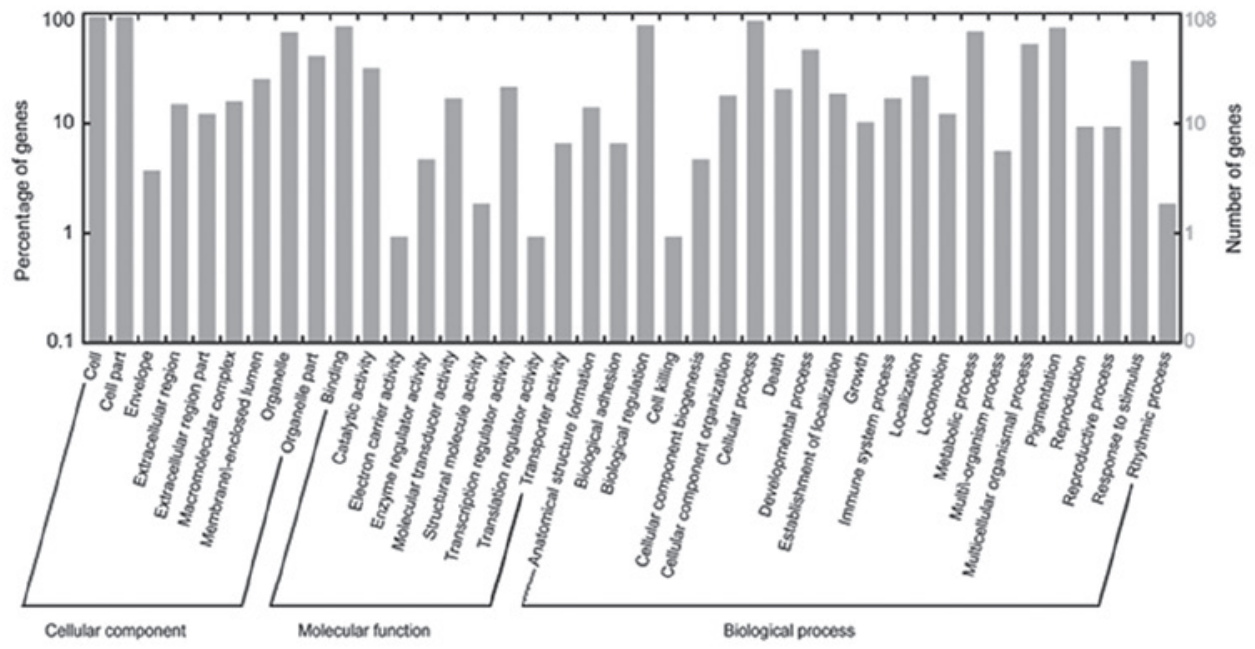

B

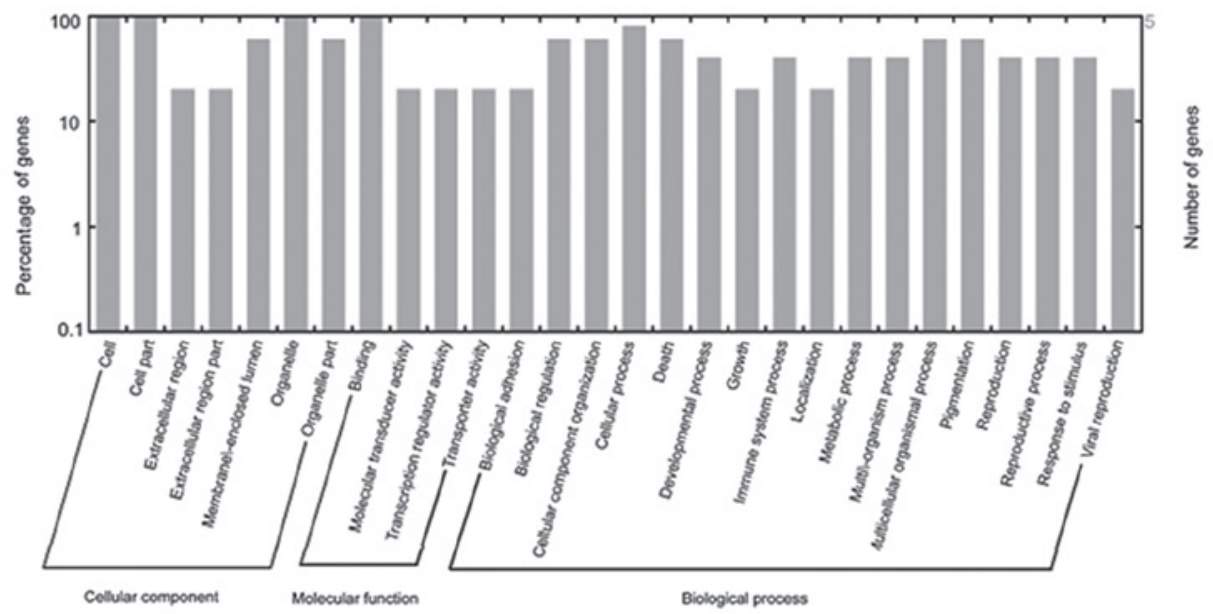

$\mathbf{C}$

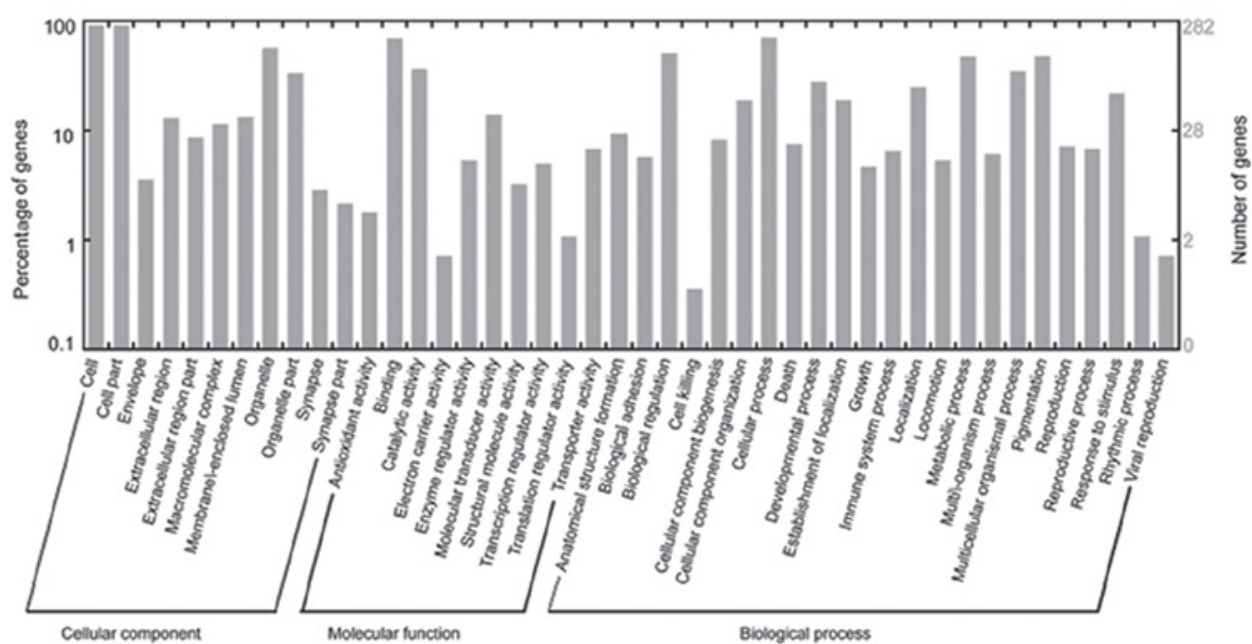

Figure 3. Functional categories, including cellular component, molecular function and biological process categories, for the (A) common DEGs, (B) propofol-specific DEGs and (C) sevoflurane-specific DEGs. DEG, differentially expressed gene.

in the functional modules for propofol-specific DEGs. Eight upregulated DEGs, including cytokine inducible SH2-containing protein (CISH), protein tyrosine phosphatase, non-receptor type 1 (PTPN1), suppressor of cytokine signaling 1 (SOCS1), interleukin 15 receptor $\alpha$ (IL15R $\alpha$ ), regulator of calcineurin 1 (RCAN1), interleukin 6 receptor (IL6R), dual specificity phosphatase 4 (DUSP4) and signal transducer and activator of transcription 3 (STAT3) were involved in the functional modules for sevoflurane-specific DEGs. In addition, seven downregulated DEGs, including carboxypeptidase $\mathrm{X}$ (M14 family), member 1 (CPXM1), fibroblast growth factor receptor 2 (FGFR2), fibronectin leucine rich transmembrane protein 3 (FLRT3), interleukin 17 receptor D (IL17RD), insulin receptor substrate 1 (IRS1), IL7 and colony stimulating 
A

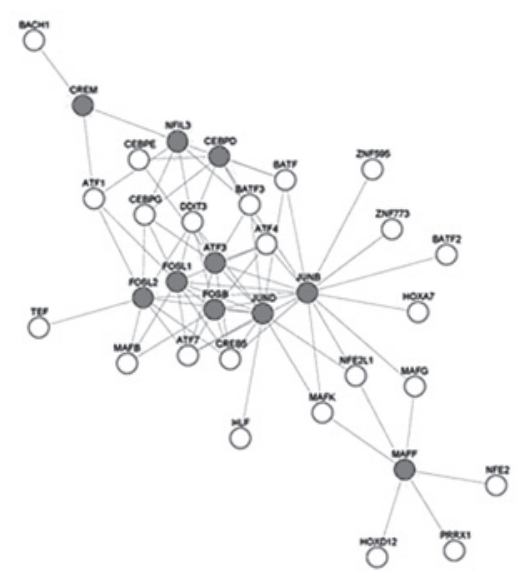

B

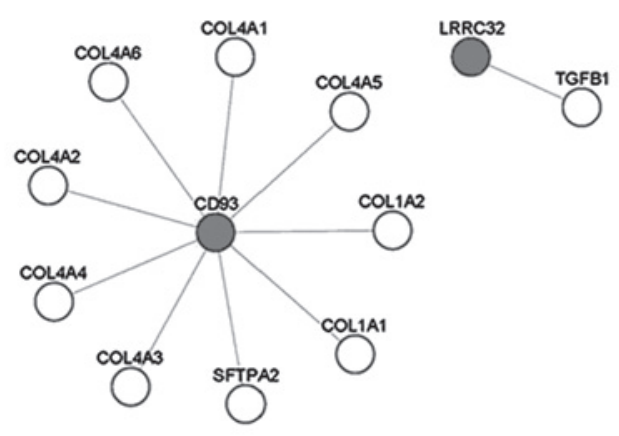

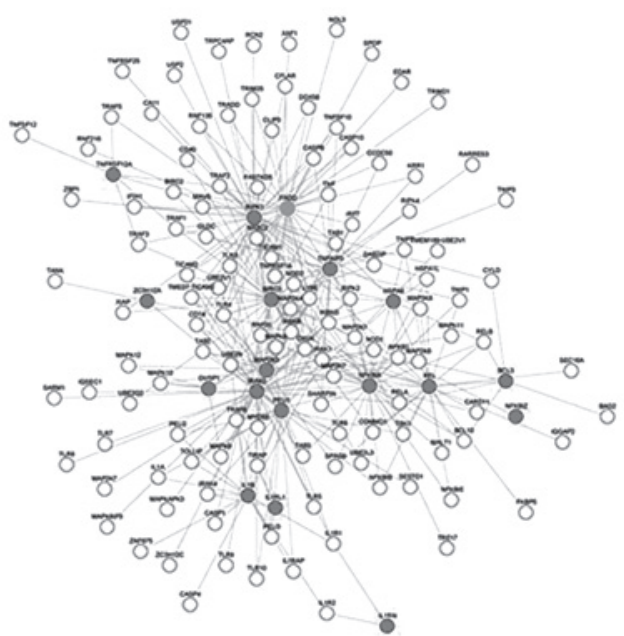

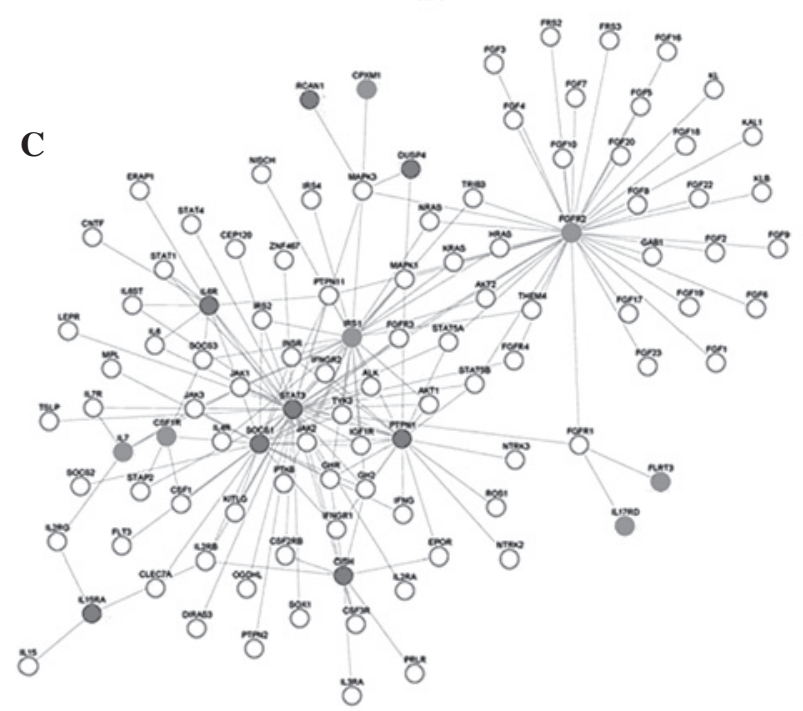

Figure 4. Functional modules of the (A) common DEGs, (B) propofol-specific DEGs and (C) sevoflurane-specific DEGs. The total functional modules for the common, propofol-specific and sevoflurane-specific DEGs were identified. Red nodes represent the upregulated DEGs; grey nodes represent the downregulated DEGs; white nodes represent the genes included in the WebGestalt database. DEG, differentially expressed gene.

factor 1 receptor (CSF1R) were also observed in the functional modules for sevoflurane-specific DEGs.

The biological processes associated with the DEGs in the modules with $\mathrm{P}<0.05$ were selected (Table I). The DEGs in these modules were involved in cellular processes, including 'regulation of transcription' and 'regulation of cellular process', which were similar to the functional annotations of the DEGs.

\section{Discussion}

Compared with on-pump CABG, the levels of TNF $\alpha$, heart-type fatty acid-binding protein and creatine kinase-MB are significantly lower in off-pump CABG, suggesting a decreased systemic inflammatory response and reduced myocardial damage $(8,37)$. However, off-pump CABG surgery can also lead to ischemic injury (8). Several studies have identified that, to a certain extent, sevoflurane and propofol are effective cardioprotective anesthetic agents $(27,38)$. In the present study, 275 common, 604 sevoflurane-specific and 15 propofol-specific DEGs were identified from expression profiles of atrial samples, which were obtained from patients receiving either the anesthetic gas sevoflurane or the intravenous anesthetic propofol prior to and following off-pump CABG surgery. Functional analysis of the modules for the common, sevoflurane-specific and propofol-specific DEGs revealed that the DEGs in the modules involved with cellular processes, including 'regulation of transcription' and 'regulation of cellular process', were similar to the functional annotations of the DEGs.

A total of 10 and 18 DEGs were present in the first and second functional modules for the common DEGs in the sevoflurane and propofol groups, including ATF3, JUND, JUNB, FOSB, MAP2K3 and TNF $\alpha$ IP3. ATF3 is a member of the activation transcription factor family of transcription factors. It has been demonstrated that ATF3 protects cardiac myocytes against doxorubicin-induced apoptosis (39). In addition, ATF3 promotes neurite outgrowth in injured neurons (40) and is important in promoting neuronal survival (41). ATF3 has been observed to interact with JUND, which may protect cells from p53-dependent senescence and apoptosis $(42,43)$. Bergman et al (44) demonstrated that nuclear extracts of cardiac fibroblasts from hypoxic rats contained Fos-related antigen 1, JUNB and FOSB, which significantly increase the transcriptional activities of cardiac 
Table I. Biological processes associated with the differentially expressed genes (DEGs) in the modules for common DEGs, propofol-specific DEGs and sevoflurane-specific DEGs.

\begin{tabular}{|c|c|c|c|c|}
\hline Module & GO-ID & P-value & Count & Description \\
\hline \multirow[t]{8}{*}{ Common 1} & 6355 & $1.668 \times 10^{-7}$ & 10 & Regulation of transcription, DNA-dependent \\
\hline & 51252 & $1.668 \times 10^{-7}$ & 10 & Regulation of RNA metabolic processes \\
\hline & 45449 & $3.545 \times 10^{-6}$ & 10 & Regulation of transcription \\
\hline & 10556 & $5.286 \times 10^{-6}$ & 10 & $\begin{array}{l}\text { Regulation of macromolecule biosynthetic } \\
\text { process }\end{array}$ \\
\hline & 10468 & $5.286 \times 10^{-6}$ & 10 & Regulation of gene expression \\
\hline & 19219 & $5.286 \times 10^{-6}$ & 10 & $\begin{array}{l}\text { Regulation of nucleobase, nucleoside, } \\
\text { nucleotide and nucleic acid metabolic process }\end{array}$ \\
\hline & 31326 & $5.286 \times 10^{-6}$ & 10 & Regulation of cellular biosynthetic process \\
\hline & 51171 & $5.286 \times 10^{-6}$ & 10 & $\begin{array}{l}\text { Regulation of nitrogen compound metabolic } \\
\text { process }\end{array}$ \\
\hline \multirow[t]{4}{*}{ Common 2} & 50794 & 0.018 & 12 & Regulation of cellular process \\
\hline & 50789 & 0.025 & 12 & Regulation of biological process \\
\hline & 65007 & 0.035 & 12 & Biological regulation \\
\hline & 48518 & 0.002 & 9 & Positive regulation of biological process \\
\hline \multirow[t]{3}{*}{ Propofol-specific } & 42116 & 0.027 & 1 & Macrophage activation \\
\hline & 2274 & 0.027 & 1 & Myeloid leukocyte activation \\
\hline & 6909 & 0.027 & 1 & Phagocytosis \\
\hline \multirow[t]{8}{*}{ Sevoflurane-specific } & 23052 & $1.590 \times 10^{-4}$ & 12 & Signaling \\
\hline & 50794 & 0.017 & 12 & Regulation of cellular process \\
\hline & 50789 & 0.024 & 12 & Regulation of biological process \\
\hline & 65007 & 0.033 & 12 & Biological regulation \\
\hline & 7165 & 0.001 & 9 & Signal transduction \\
\hline & 23033 & 0.002 & 9 & Signaling pathway \\
\hline & 23060 & 0.002 & 9 & Signal transmission \\
\hline & 23046 & 0.002 & 9 & Signaling process \\
\hline
\end{tabular}

GO, gene ontology; Count, number of DEGs involved in the identified biological processes.

fibroblasts (44). Furthermore, discrete activator protein-1 components, including JUNB and FOSB are induced by oxidative stress and mediate the transcription and translation of matrix metalloproteinase 2 , which is important in the response of the heart following cardiac ischemic-reperfusion injury (45). MAP2K3 belongs to the MAP kinase family and is involved in the MAPK-mediated signaling cascade, which is possibly important in the pathogenesis of cardiac and vascular disease $(46,47)$. Transfection experiments have confirmed that p38-MAPK is involved in cardiac myocyte apoptosis (48). Previous studies have demonstrated that single nucleotide polymorphisms in TNF $\alpha$ IP3 are associated with an increased risk of coronary artery disease in type 2 diabetes and of left ventricular hypertrophy in hypertensive patients $(49,50)$. The activation of nuclear factor- $\kappa \mathrm{B}$ can be inhibited by overexpression of TNF $\mathrm{IP} 3$ to attenuate cardiac myocyte hypertrophy $(51,52)$.

In the functional modules for propofol-specific DEGs, two upregulated DEGs, CD93 and LRRC32, were involved. A total of eight upregulated DEGs (CISH, PTPN1, SOCS1, IL15R $\alpha$, RCAN1, IL6R, DUSP4 and STAT3) and seven downregulated DEGs (CPXM1, FGFR2, FLRT3, IL17RD,
IRS1, IL7 and CSF1R) were identified in the functional modules for sevoflurane-specific DEGs. A study by Lucchinetti et al (28), STAT3 was found to be associated with the granulocyte-colony stimulating factor survival pathway. In the present study, the function of 'response to wounding', which involved 72 DEGs, was identified as the most significant GO term of the 879 DEGs in the sevoflurane group and the 'response to organic substance' function, involving 45 DEGs, was the most significant GO term of the 290 DEGs in the propofol group. The sevoflurane-specific DEGs in the interactive functional modules were also associated significantly with the 'regulation of cellular process' Go term.

However, the genes involved in the molecular mechanisms underlying sevoflurane and propofol cardioprotective effects requires further confirmation at the gene and protein levels using reverse transcription quantitative polymerase chain reaction and western blot analyses. Therefore, subsequent investigations aim to include a cell culture system or animal models to confirm the results of the present study.

In conclusion, sevoflurane and propofol may synergistically reduce myocardial reperfusion injury in patients 
undergoing $\mathrm{CABG}$ surgery, since similarity was observed in the changes in expression induced by sevoflurane and propofol. However, the underlying molecular mechanisms for the effects of anaesthetic agents in cardioprotection require further investigation and confirmation.

\section{References}

1. Shekar PS: Cardiology patient page. On-pump and off-pump coronary artery bypass grafting. Circulation 113: e51-e52, 2006.

2. Ehsan A, Shekar P and Aranki S: Innovative surgical strategies: Minimally invasive CABG and off-pump CABG. Curr Treat Options Cardiovasc Med 6: 43-51, 2004.

3. Palmerini T, Biondi-Zoccai G, Riva DD, et al: Risk of stroke with percutaneous coronary intervention compared with on-pump and off-pump coronary artery bypass graft surgery: Evidence from a comprehensive network meta-analysis. Am Heart J 165: 910-917, 2013.

4. Harskamp RE, Lopes RD, Baisden CE, De Winter RJ and Alexander JH: Saphenous vein graft failure after coronary artery bypass surgery: pathophysiology, management and future directions. Ann Surg 257: 824-833, 2013.

5. Bakaeen FG, Chu D, Kelly RF, et al: Performing Coronary Artery Bypass Grafting Off-Pump May Compromise Long-Term Survival in a Veteran Population. Ann Thorac Surg 95: 1952-1958, 2013.

6. Moller CH, Penninga L, Wetterslev J, Steinbruchel DA and Gluud C: Off-pump versus on-pump coronary artery bypass grafting for ischaemic heart disease. Cochrane Database Syst Rev 3: CD007224, 2012.

7. Nathoe HM, Van Dijk D, Jansen EW, et al: A comparison of on-pump and off-pump coronary bypass surgery in low-risk patients. N Engl J Med 348: 394-402, 2003.

8. Orhan G, Sargin M, Senay S, et al: Systemic and myocardial inflammation in traditional and off-pump cardiac surgery. Tex Heart Inst J 34: 160-165, 2007.

9. Selvanayagam JB, Petersen SE, Francis JM, et al: Effects of off-pump versus on-pump coronary surgery on reversible and irreversible myocardial injury: a randomized trial using cardiovascular magnetic resonanceimaging and biochemical markers. Circulation 109: 345-350, 2004.

10. Menasché P: The systemic factor: the comparative roles of cardiopulmonary bypass and off-pump surgery in the genesis of patient injury during and following cardiac surgery. Ann Thorac Surg 72: S2260-S2265; discussion S2265-S2266, S2267-S2270, 2001.

11. Tomic V, Russwurm S, Moller E, et al: Transcriptomic and proteomic patterns of systemic inflammation in on-pump and off-pump coronary artery bypass grafting. Circulation 112: 2912-2920, 2005

12. Brown J,Hernandez F, Beaulieu P, Clough R and Whited C, et al: Off-pump coronary artery bypass does not influence biomarkers of brain injury, but does exacerbate the systemic inflammatory response. J Clin Cell Immunik S2, 001, 2011.

13. Frassdorf J, De Hert S and Schlack W: Anaesthesia and myocardial ischaemia/reperfusion injury. Br J Anaesth 103 . 89-98, 2009.

14. Varadarajan SG, An J, Novalija E and Stowe DF: Sevoflurane before or after ischemia improves contractile and metabolic function while reducing myoplasmic $\mathrm{Ca}\left({ }^{2+}\right)$ loading in intact hearts. Anesthesiology 96: 125-133, 2002

15. Preckel B, Schlack W, Comfère T, Obal D, Barthel H and Thämer V: Effects of enflurane, isoflurane, sevoflurane and desflurane on reperfusion injury after regional myocardial ischaemia in the rabbit heart in vivo. Br J Anaesth 81: 905-912, 1998.

16. Lin E and Symons JA: Volatile anaesthetic myocardial protection: a review of the current literature. HSR Proc Intensive Care Cardiovasc Anesth 2: 105-109, 2010.

17. Garcia C, Julier K, Bestmann L, et al: Preconditioning with sevoflurane decreases PECAM-1 expression and improves one-year cardiovascular outcome in coronary artery bypass graft surgery. Br J Anaesth 94: 159-165, 2005.

18. Kawamura T, Kadosaki M, Nara N, et al: Effects of sevoflurane on cytokine balance in patients undergoing coronary artery bypass graft surgery. J Cardiothorac Vasc Anesth 20: 503-508, 2006.
19. Huang Z, Zhong X, Irwin MG, et al: Synergy of isoflurane preconditioning and propofol postconditioning reduces myocardial reperfusion injury in patients. Clin Sci (Lond) 121: 57-69, 2011.

20. Corcoran TB, Engel A, Sakamoto H, O'shea A, O'callaghan-Enright S and Shorten GD: The effects of propofol on neutrophil function, lipid peroxidation and inflammatory response during elective coronary artery bypass grafting in patients with impaired ventricular function. Br J Anaesth 97: 825-831, 2006

21. Sun HY, Xue FS, Xu YC, et al: Propofol improves cardiac functional recovery after ischemia-reperfusion by upregulating nitric oxide synthase activity in the isolated rat hearts. Chin Med J (Engl) 122: 3048-3054, 2009.

22. Wang HY, Wang GL, Yu YH and Wang Y: The role of phosphoinositide-3-kinase/Akt pathway in propofol-induced postconditioning against focal cerebral ischemia-reperfusion injury in rats. Brain Res 1297: 177-184, 2009.

23. Kottenberg E, Thielmann M, Bergmann L, et al: Protection by remote ischemic preconditioning during coronary artery bypass graft surgery with isoflurane but not propofol - a clinical trial. Acta Anaesthesiol Scand 56: 30-38, 2012.

24. Zaugg M, Lucchinetti E, Spahn DR, Pasch T, Garcia C and Schaub MC: Differential effects of anesthetics on mitochondrial K(ATP) channel activity and cardiomyocyte protection. Anesthesiology 97: 15-23, 2002.

25. Müllenheim J, Ebel D, Frässdorf J, Preckel B, Thämer V and Schlack W: Isoflurane preconditions myocardium against infarction via release of free radicals. Anesthesiology 96: 934-940, 2002

26. Heusch G, Boengler K and Schulz R: Cardioprotection: nitric oxide, protein kinases and mitochondria. Circulation 118: 1915-1919, 2008

27. He W, Zhang FJ, Wang SP, Chen G, Chen CC and Yan M: Postconditioning of sevoflurane and propofol is associated with mitochondrial permeability transition pore. J Zhejiang Univ Sci B 9: 100-108, 2008.

28. Lucchinetti E, Hofer C, Bestmann L, et al: Gene regulatory control of myocardial energy metabolism predicts postoperative cardiac function in patients undergoing off-pump coronary artery bypass graft surgery: inhalational versus intravenous anesthetics. Anesthesiology 106: 444-457, 2007.

29. Fujita A, Sato JR, Rodrigues Lde O, Ferreira CE and Sogayar MC: Evaluating different methods of microarray data normalization. BMC Bioinformatic 7: 469, 2006.

30. Smyth GK: Limma: linear models for microarray data. In: Bioinformatics and Computational Biology Solutions using $\mathrm{R}$ and Bioconductor. Gentleman R, Carey, V, Dudoit S, Irazarry R and Huber W (eds). Springer, New York, NY, pp397-420, 2005.

31. Benjamini Y and Hochberg Y: Controlling the false discovery rate: a practical and powerful approach to multiple testing. J R Stat Soc B 1: 289-300, 1995.

32. Huang Da W, Sherman BT and Lempicki RA: Systematic and integrative analysis of large gene lists using DAVID Bioinformatics Resources. Nature Protoc 4: 44-57, 2009.

33. Tatusov RL, Natale DA, Garkavtsev IV, et al: The COG database: new developments in phylogenetic classification of proteins from complete genomes. Nucleic Acids Res 29: 22-28, 2001.

34. Altschul SF, Gish W, Miller W, Myers EW and Lipman DJ: Basic local alignment search tool. J Mol Biol 215: 403-410, 1990.

35. Zhang B, Kirov S and Snoddy J: WebGestalt: an integrated system for exploring gene sets in various biological contexts. Nucleic Acids Res 33: W741-W748, 2005.

36. Shi G, Zhang L and Jiang T: MSOAR 2.0: Incorporating tandem duplications into ortholog assignment based on genome rearrangement. BMC Bioinformatics 11: 10, 2010.

37. Malik V, Kale SC, Chowdhury UK, Ramakrishnan L, Chauhan S and Kiran U: Myocardial injury in coronary artery bypass grafting: On-pump versus off-pump comparison by measuring heart-type fatty-acid-binding protein release. Tex Heart Inst J 33: 321-327, 2006.

38. Mathur S, Farhangkhgoee P and Karmazyn M: Cardioprotective effects of propofol and sevoflurane in ischemic and reperfused rat hearts: role of K(ATP) channels and interaction with the sodium-hydrogen exchange inhibitor HOE 642 (cariporide). Anesthesiology 91: 1349-1360, 1999.

39. Nobori $\mathrm{K}$, Ito $\mathrm{H}$, Tamamori-Adachi $\mathrm{M}$, et al: ATF3 inhibits doxorubicin-induced apoptosis in cardiac myocytes: a novel cardioprotective role of ATF3. J Mol Cell Cardiol 34: 1387-1397, 2002. 
40. Seijffers R, Allchorne AJ and Woolf CJ: The transcription factor ATF-3 promotes neurite outgrowth. Mol Cell Neurosci 32: $143-154,2006$

41. Francis JS, Dragunow M and During MJ: Over expression of ATF-3 protects rat hippocampal neurons from in vivo injection of kainic acid. Brain Res Mol Brain Res 124: 199-203, 2004.

42. Chu HM, Tan Y, Kobierski LA, Balsam LB and Comb MJ: Activating transcription factor-3 stimulates 3',5'-cyclic adenosine monophosphate-dependent gene expression. Mol Endocrinol 8: 59-68, 1994.

43. Weitzman JB, Fiette L, Matsuo K and Yaniv M: JunD protects cells from p53-dependent senescence and apoptosis. Mol Cell 6: $1109-1119,2000$

44. Bergman MR, Cheng S, Honbo N, Piacentini L, Karliner JS and Lovett DH: A functional activating protein 1 (AP-1) site regulates matrix metalloproteinase 2 (MMP-2) transcription by cardiac cells through interactions with JunB-Fra1 and JunB-FosB heterodimers. Biochem J 369: 485-496, 2003.

45. Alfonso-Jaume MA, Bergman MR, Mahimkar R, et al: Cardiac ischemia-reperfusion injury induces matrix metalloproteinase-2 expression through the AP-1 components FosB and JunB. Am J Physiol Heart Circ Physiol 291: H1838-H1846, 2006.

46. Kumar S, Boehm J and Lee JC: p38 MAP kinases: key signalling molecules as therapeutic targets for inflammatory diseases. Nat Rev Drug Discov 2: 717-726, 2003.
47. Muslin AJ: MAPK signalling in cardiovascular health and disease: molecular mechanisms and therapeutic targets. Clin Sci (Lond) 115: 203-218, 2008.

48. Wang Y, Huang S, Sah VP, et al: Cardiac muscle cell hypertrophy and apoptosis induced by distinct members of the p38 mitogen-activated protein kinase family. J Biol Chem 273: 2161-2168, 1998.

49. Boonyasrisawat W, Eberle D, Bacci S, et al: Tag polymorphisms at the A20 (TNFAIP3) locus are associated with lower gene expression and increased risk of coronary artery disease in type 2 diabetes. Diabetes 56: 499-505, 2007.

50. Xue H, Wang SX, Wang XJ, et al: Variants of tumor necrosis factor-induced protein 3 gene are associated with left ventricular hypertrophy in hypertensive patients. Chin Med J (Engl) 124: 1498-1503, 2011.

51. De Keulenaer GW, Wang Y, Feng Y, et al: Identification of IEX-1 as a biomechanically controlled nuclear factor-kappaB target gene that inhibits cardiomyocyte hypertrophy. Circ Res 90: 690-696, 2002.

52. Hirotani S, Otsu K, Nishida K, et al: Involvement of nuclear factor-kappaB and apoptosis signal-regulating kinase 1 in G-protein-coupled receptor agonist-induced cardiomyocyte hypertrophy. Circulation 105: 509-515, 2002. 